\section{AB0552 25 HYDROXY-VITAMIN D LEVEL IN SYSTEMIC LUPUS ERYTHEMATOSUS: IS IT RELATED TO DISEASE ACTIVITY AND LUPUS NEPHRITIS?}

Alshaimaa Rezk Lotfy Rezk Alnaggar, Ahmed Fayed, Ahmed Soliman. Cairo University, Faculty Of Medicine, Kasr Al Ainy, Internal Medicine, Cairo, Egypt

Background: Systemic lupus erythematosus (SLE) is a chronic autoimmune disease. SLE is attributed to different autoimmune mechanisms leading to the production of several autoantibodies and formation of immune complexes with subsequent organ damage. 25 hydroxy-vitamin D3 $(25(\mathrm{OH}) \mathrm{D} 3)$ plays a significant role in immune system regulation and is an important immunomodulatory hormone involved in various biochemical reactions.

Objectives: Are to assess the levels of $25(\mathrm{OH}) \mathrm{D} 3$ in patients with SLE and to investigate the relationship between $25(\mathrm{OH}) \mathrm{D} 3$ levels and disease activity in patients with and without lupus nephritis.

Methods: 300 subjects were included in the study and were divided into 3 groups: group 1 consisted of 100 patients with SLE and lupus nephritis (LN), group 2 consisted of 100 patients free from LN and group 3 consisted of 100 healthy volunteers as a control group. All patients fullfilled the American College of Rheumatology criteria for diagnosis of SLE and were recruited from Internal Medicine department and out-patient clinics, Cairo University Hospital. Exclusion criteria included diseases and drugs which affect the endothelial function such as: smoking, diabetes mellitus, essential hypertension, and coronary artery disease and drugs such as nitrates, hypolipidaemic drugs and aspirin. Disease activity was evaluated by systemic lupus erythematosus disease activity index (SLEDAI). 25(OH) D3 was measured using ELISA. 25(OH)D3 level $\geq 30 \mathrm{ng} / \mathrm{ml}$ was considered as recommended, $<30 \mathrm{ng} / \mathrm{ml}$ was considered as vitamin $D$ insufficiency and $<25 \mathrm{ng} / \mathrm{ml}$ was considered vitamin $D$ deficiency.

Results: The mean value of $25(\mathrm{OH}) \mathrm{D} 3$ was significantly lower in patients with lupus nephritis $(17.1 \pm 5.5 \mathrm{ng} / \mathrm{ml})$ and those without lupus nephritis $(16.6 \pm 5.9 \mathrm{ng} / \mathrm{ml})$ than in controls $(36.7 \pm 3.3 \mathrm{ng} / \mathrm{ml})$ ( $p$-value 0.001$)$. Serum 25(OH)D3 level was inversely correlated with the duration of SLE disease ( $r-0.676, p$-value $<0.001)$ and with the duration of lupus nephritis $(r$ $0.363, p$-value $<0.001)$. There was strong negative correlation between 25 $(\mathrm{OH}) \mathrm{D} 3$ levels and serum urea, creatinine, ANA titre, anti-dsDNA titre, C3 and C4. 25(OH)D3 level was negatively correlated with, ESR, CRP and SLEDAI score $(r=-0.811, p$-value $=0.001)$. There was also highly significant negative correlation between 25(OH)D3 level and prednisolone dose, hydroxychloroquine, cyclophosphamide and mycophenolate mofetil but not with NSAIDs.

Conclusion: 25(OH)D3 levels are markedly lower in patients with SLE. Low 25(OH)D3 levels are significantly correlated with disease activity parameters, disease duration and disease treatment.

\section{REFERENCES}

[1] Mok CC. Emerging biological therapies for systemic lupus erythematosus. Expert Opin Emerg Drugs. 2014;19(2):303-22.

[2] Yeap SS, Othman AZ, Zain AA, Chan SP. Vitamin D levels: its relationship to bone mineral density response and disease activity in premenopausal Malaysian systemic lupus erythematosus patients on corticosteroids. Int $\mathrm{J}$ Rheum Dis. 2012;15(1):17-24.

[3] Borges MC, Martini LA, Rogero MM. Current perspectives on vitamin D, immune system, and chronic diseases. Nutrition. 2011;27(4):399-404.

\section{Vit. D level in studied groups}

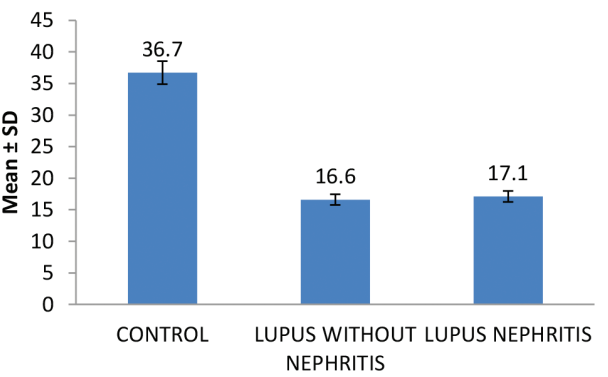

Figure 1. Level of serum 25(OH)D3 in patients with SLE and controls:

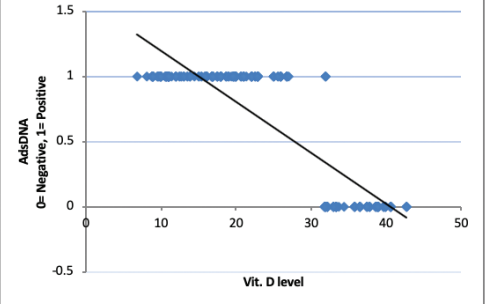

(a) Correlation between AdsDNA with Vit. $D$ level.

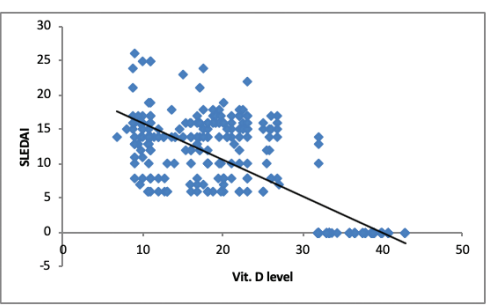

(b) Correlation bełween SLEDAI with Vit. D level.

Figure 2. Correlation between 25(OH)D3 levels and SLEDAI and anti-ds DNA

Table 1. 25(OH)D3 levels and SLEDIA in patients with and without lupus nephritis

\begin{tabular}{lccccc}
\hline SLEDAl grade & \multicolumn{4}{c}{ 25(OH)D3 level } \\
\cline { 2 - 5 } & \multicolumn{1}{c}{ WITHOUT LUPUS NEPHRITIS } & \multicolumn{1}{c}{ LUPUS NEPHRITIS } & \multirow{2}{*}{ P- value } \\
\cline { 2 - 5 } & $<\mathbf{3 0}$ & $\mathbf{3 0}$ & $<\mathbf{3 0}$ & $\mathbf{3 0}$ & \\
\hline Mild (1-5) & $0(0.0 \%)$ & $0(0.0 \%)$ & $0(0.0 \%)$ & $0(0.0 \%)$ & $0.001^{* *}$ \\
Moderate (6-12) & $4(100.0 \%)$ & $0(0.0 \%)$ & $69(98.6 \%)$ & $1(1.4 \%)$ & \\
Severe (13-20) & $94(97.9 \%)$ & $2(2.1 \%)$ & $30(100.0 \%)$ & $0(0.0 \%)$ & \\
\hline
\end{tabular}

Disclosure of Interests: None declared

DOI: 10.1136/annrheumdis-2019-eular.158

\section{\begin{tabular}{|l|l}
\hline AB0553 STUDY OF ANXIETY AND DEPRESSION IN PATIENTS \\
\hline
\end{tabular} WITH SYSTEMIC LUPUS ERYTHEMATOSUS (SLE)}

Marta Rojas-Giménez ${ }^{1}$, Stack John², James Galloway², Natalia Mena-Vázquez ${ }^{1}$, Clara Fuego-Varela ${ }^{1}$, Antonio Fernandez-Nebro ${ }^{1} .{ }^{1}$ Rheumatology Service of the Regional University Hospital of Malaga (HRUM), Biomedical Research Institute of Malaga (IBIMA), University of Málaga., Malaga, Spain; ${ }^{2}$ KING'S COLLEGE HOSPITAL NHS FOUNDATION TRUST, London, United Kingdom Objectives: To study the prevalence of anxiety and depression in a cohort of patients with SLE at King's College Hospital in London,as well as its relationship with characteristics of disease and treatments

Methods: Cross-sectional study of a cohort of patients with SLE.All patients with SLE who had been included in the IMPARTs project(Integrating Mental and Physical healthcare:Research,Training and Services) were recruited between 2012-2016.All patients filled out the following questionnaires on a tablet device:PHQ9(Patient Health Questionnaire) (Scores of 10 or more have an sensitivity and specifity close to $90 \%$ for major depression) and GAD-7(7-item Generalized Anxiety Disorder)(Scores of 10 or more have an sensitivity and specifity close to $89 \%$ and $82 \%$ for GAD).Variables:demographic, duration of the disease(months)and the profile of autoantibodies.Previous history of depression or anxiety. SLEDAl,treatments,ESR, CPR,C3 and C4.Statistical analysis:descriptive, bivariate using T-Student and $\chi 2$. 